\title{
Association between severity of depression and clinico-biochemical markers of polycystic ovary syndrome
}

\author{
Behnaz Enjezab ${ }^{1}$, Maryam Eftekhar ${ }^{2,3}$, Akram Ghadiri-Anari ${ }^{4}$
}

${ }^{1}$ Ph.D. in Reproductive Health, Associate Professor, Research Center for Nursing and Midwifery Care, Department of Midwifery, Faculty of Nursing \& Midwifery, Shahid Sadoughi University of Medical Sciences, Yazd, Iran

${ }^{2}$ M.D., Gynecologist, Associate Professor, Research and Clinical Center for Infertility, Yazd Reproductive Sciences Institute, Shahid Sadoughi University of Medical Sciences, Yazd, Iran

3 Abortion Research Center, Yazd Reproductive Sciences Institute, Shahid Sadoughi University of Medical Sciences, Yazd, Iran

${ }^{4}$ M.D., Endocrinologist, Associate Professor, Diabetes Research Center, Shahid Sadoughi University of Medical Sciences, Yazd, Iran

\section{Type of article: Original}

\begin{abstract}
Background: Polycystic ovary syndrome (PCOS), as the most common endocrine disorder among reproductiveaged women may lead to psychological morbidity.

Objectives: The present study set out to evaluate the severity of depression in PCOS and non-PCOS women and to evaluate correlation between depression score with body mass index, HOMA-IR, and testosterone level in every group (PCOS and non-PCOS women) in Yazd province.

Methods: In this cross-sectional study, 62 women with PCOS and 61 non- PCOS subjects (20 to 40 years) who were attending Imam-Ali Clinic in Yazd, Iran from September 2014 through March 2015 participated. These persons completed the Beck Depression Short Inventory (BDI-S) Questionnaire. Frequency of depression in PCOD and healthy persons and correlation between depression severity with body mass index, HOMA-IR, testosterone level in PCOS and non- PCOS women were evaluated by ANOVA test and independent-samples ttest and Pearson correlation. P-value of less than 0.05 was considered as statistically significant. Data analyses were performed using SPSS version 16.

Results: Of 62 PCOS subjects, 40 (64.5\%) were verified positive for depression, determined by the BDI-S Questionnaire. In the control group, $60.7 \%$ had depression. Mean \pm SE score of depression for PCOS and nonPCOS women were $7.47 \pm 5.54$ and $7.57 \pm 5.77$ respectively. There was no statistically significant correlation between body mass index, HOMA-IR, testosterone level with depression score in either group separately or together.

Conclusions: We found considerable amounts of depression in our population. There is no correlation between body mass index, HOMA-IR, and testosterone level with depression score in our study.

Keywords: Polycystic ovary syndrome, Depression, Insulin resistance, Testosterone, Hirsutism
\end{abstract}

\section{Introduction}

Polycystic ovary syndrome (PCOS) is characterized by irregular menses, hyperandrogenism, and polycystic ovaries. Its clinical presentations include oligomenorrhea or amenorrhea, hirsutism, acne, androgenic alopecia, obesity, and infertility. It is as one of the most common endocrine/metabolic disorders among women in reproductive age (1-3). Women with PCOS are more likely to have increased risk of diabetes mellitus, dyslipidemia, and metabolic disorders associated with insulin resistance (4). This chronic condition with long-term complications may lead to reduced quality of life, impatience, irritability, depression, and anxiety. The prevalence of depression in women with

\section{Corresponding author:}

Associate Professor Dr. Akram Ghadiri-Anari, Diabetes Research Center, Shahid Sadoughi University of Medical Sciences, Yazd, Iran. Tel: +98.3531833434, Fax:+98.3538224100, Email: ghadiriam@yahoo.com

Received: April 24, 2017, Accepted: August 06, 2017, Published: November 2017

iThenticate screening: July 25, 2017, English editing: September 22, 2017, Quality control: October 15, 2017

This article has been reviewed / commented by three experts

(C) 2017 The Authors. This is an open access article under the terms of the Creative Commons Attribution-NonCommercialNoDerivs License, which permits use and distribution in any medium, provided the original work is properly cited, the use is non-commercial and no modifications or adaptations are made. 
PCOS is high and varies from $28 \%$ to $64 \%(5,6)$. Correct diagnosis and treatment of PCOS can prevent future metabolic, endocrine, and cardiovascular outcomes and decrease psychological stresses $(5,7)$. Women with hyperandrogenism syndrome are at increased risk for mood disorders that may be due to the relationship between androgen levels and depression (8). Some studies reported a significant increase in prevalence of depression among PCOS women compared with the controls $(3,9)$. Ragson and others find a significant relationship between depression and PCOS markers such as insulin resistance and body mass index (BMI), but association between depression and hirsutism, acne, alopecia, and irregular menses were not mentioned (3). Weiner showed high prevalence of depression in PCOS patients related to influences of testosterone on adult female behavior (10). It seems that the clinician should pay attention to the cultural background of their PCOS patients especially in view of the factors influencing psychological well-being (11). Considering the facts that PCOS patients are young women living in their active life period, and the high prevalence of depression in these young persons, the effects of depression on their lives, and the necessity for studies in different ethnic groups due to the effect of cultural background together with lack of data on the relationship between biochemical markers of PCOS and severity of depression in Yazd province, this study was designed to evaluate this problem.

\section{Material and Methods}

\subsection{Study populations and design}

This study was a cross-sectional study of 62 women with PCOS and 61 non- PCOS subjects (aged 20 to 40 years) consecutively came to Imam-Ali Clinic in Yazd, Iran from September 2014 through March 2015. Non-random simple sampling was used and a researcher examined all subjects.

\subsection{Selection criteria}

\subsubsection{Inclusion criteria}

Participants were included in the PCOS group if they fulfilled the revised Rotterdam criteria for PCOS diagnosis, which are presence of two of the following three features (1) Oligomenorrhea; (2) Clinical and/or biochemical hyperandrogenism; and (3) Polycystic ovaries (4). Oligomenorrhea was defined as below eight cycles per year during an extended period of time when not on oral contraceptive pills. Clinical hyperandrogenism included hirsutism and or significant acne warranting treatment. Biochemical hyperandrogenism included elevated total testosterone, free testosterone, or dehydroepiandrosterone sulfate. Polycystic ovaries were defined as the presence of $\geq 12$ follicles in each ovary with each follicle measuring 2 to $9 \mathrm{~mm}$ in diameter and/or an increased ovarian volume $(>10 \mathrm{~mL})$ in sonography performed by a radiologist (4). The control group consisted of sixty-one healthy subjects with age matched with PCOS patients. Controls were selected from the healthy persons who came with patients (PCOS and other patients) and had regular menses and no evidence of hyperandrogenism.

\subsubsection{Exclusion criteria}

Persons with the following criteria were excluded from study: Any known chronic diseases such as diabetes mellitus, cirrhosis, thyroid disorders, other cause of oligomenorrhea and hyperandrogenism (Congenital adrenal hyperplasia, prolactinoma, Cushing's syndrome and etc.), history of severe stress such as the death of a family member in the past six months, hormone therapy during the last three months, severe depression, antidepressant or corticosteroid use, alcohol consumption, drug addiction, infertility, pregnancy or lactation in the last six months. Also, women who had stressful life events such as loss of a family member or divorce in the past two years were excluded from the study.

\subsection{Data Collection}

All patients completed the Beck Depression Short Inventory (BDI-S) Questionnaire. This instrument for assessing depressive symptoms includes 13 items. Each item has a 4-point scale, where $0=$ rarely or none of the time (less than 1 day), 1 = some or a little of the time (1-2 days), 2 = occasionally or a moderate amount of time (3-4 days), 3

$=$ most or all of the time (5-7 days). Score range is from 0 to 39 and a higher score reflects greater symptoms of depression. The following have been suggested to interpret this: No depression is 0-4 points, Minimal 5-7 points; Moderate 8-15points and severe depression $\geq 16$ (12). Also, study participants completed a comprehensive questionnaire addressing various aspects of their demographics, medical history, health behaviors including exercise, and psychological adjustment. Body Mass Index (BMI) were calculated as weight $(\mathrm{kg}) / \mathrm{squared}$ height (meters) (13). All subjects were tested for fasting blood sugar (FBS), fasting insulin and total testosterone. Plasma glucose level were measured by glucose oxidase method and sample aliquots were stored at $-80{ }^{\circ} \mathrm{C}$ for assessment of insulin (I, Millipore RIA, St. Charles MO, sensitivity: $2 \mathrm{uU} / \mathrm{ml}$ ) and total testosterone (T, IRA DSL Webster TX. sensitivity: $5 \mathrm{ng} / \mathrm{dl}$ ). Insulin resistance was calculated according to HOMA index. Homeostasis Model Assessment of IR (HOMA-IR) has proved to be a robust tool for the surrogate assessment of insulin resistance (14). It is 
http://www.ephysician.ir

calculated (15) multiplying fasting plasma insulin (FPI) by fasting plasma glucose (FPG), then dividing by the constant 22.5: HOMA-IR $=($ Insulin $\times$ FPG $) / 22.5$. The HOMA-IR above 1.775 defined insulin resistance in the Iranian population (16).

\subsection{Ethical considerations}

The ethics committee of the Shahid Sadoughi University of Medical Sciences, Yazd, Iran approved the study protocol (Code: 1394.178). All participants gave informed consent to participate in this study. Also, their visit and laboratory tests were free of charge. The women's anonymity was respected.

\subsection{Statistical analysis}

Statistical analyses were performed using SPSS 16.0 (SPSS Inc., Chicago, ILL, USA). Data are presented as mean \pm $\mathrm{SD}$ or as frequency with percentages. ANOVA test and independent-samples t-test was used for normal distribution parameters analysis. The Pearson correlation evaluated the relationship between parameters. $\mathrm{P}$-value less than 0.05 were considered as statistically significant.

\section{Results}

This study included 62 women with PCOS and 61 non-PCOS subjects, age from 20 to 40 years old. There was statistically significant difference in mean of BMI between PCOS and the other group ( $p=0.002)($ Table 1). Demographic characteristics of participants were presented in Table 1. Our results showed that the overall depression score in two groups was 7.47 \pm 5.54 and 7.57 \pm 5.77 PCOS and non-PCOS respectively ( $\mathrm{p}=0.91$ ). In total, $64.5 \%$ of PCOS subjects and $60.7 \%$ of control group had some degree of depression that is not significant (Table 2).

Table 1. Demographic characteristics of participants in PCOS and non-PCOS group

\begin{tabular}{|l|l|l|l|}
\hline Variables & PCOs group $(\mathrm{n}=62)$ & Non-PCOs group $(\mathrm{n}=61)$ & p-value* \\
\hline Age (years) & $29.97 \pm 6.85$ & $29.49 \pm 7.44$ & 0.71 \\
\hline Gravid (n) & $1.15 \pm 1.34$ & $1.42 \pm 1.54$ & 0.28 \\
\hline Parity (n) & $1.07 \pm 0.90$ & $1.18 \pm 1.34$ & 0.20 \\
\hline Abortion (n) & $0.26 \pm 0.65$ & $0.25 \pm 0.56$ & 0.91 \\
\hline Systolic blood pressure (mmHg) & $117.42 \pm 10.07$ & $117.18 \pm 6.66$ & 0.87 \\
\hline Diastolic blood pressure (mmHg) & $75.42 \pm 9.27$ & $76.43 \pm 6.56$ & 0.48 \\
\hline PCO duration (months) & $56.76 \pm 66.76$ & 0 & - \\
\hline Menstrual days (days) & $7.49 \pm 2.86$ & $6.95 \pm 1.49$ & 0.72 \\
\hline Menstrual cycle (days) & $40.94 \pm 1.90$ & $28.75 \pm 3.12$ & 0.57 \\
\hline BMI & $29.16 \pm 6.56$ & $25.66 \pm 5.48$ & 0.002 \\
\hline
\end{tabular}

*Comparison of two groups performed by independent-samples t- test

Table 2. Comparison of evaluated variables in two groups

\begin{tabular}{|l|l|l|l|}
\hline Variables & $\begin{array}{l}\text { PCOS group } \\
(\mathrm{n}=62)\end{array}$ & $\begin{array}{l}\text { Non-PCOS group } \\
(\mathrm{n}=61)\end{array}$ & -value* \\
\hline Depression Score (Mean \pm SE) & $7.47 \pm 5.54$ & $7.57 \pm 5.77$ & 0.91 \\
\hline No depression (Normal) n (\%) & $22(35.5)$ & $24(39.3)$ & 0.55 \\
\hline Mild depression n (\%) & $17(27.4)$ & $15(24.6)$ & 0.59 \\
\hline Moderate depression n (\%) & $17(27.4)$ & $12(19.7)$ & 0.32 \\
\hline Severe depression n (\%) & $6(9.7)$ & $10(16.4)$ & 0.41 \\
\hline Insulin resistance (HOMA -IR) & $2.36 \pm 1.69$ & $1.70 \pm 1.21$ & 0.053 \\
\hline Testosterone level (ng/dl) & $68 \pm 28$ & $56 \pm 18$ & 0.025 \\
\hline
\end{tabular}

*Independent-samples t-test was used.

Also, comparison of depression severity in PCOS and non-PCOS based on insulin resistance (Normal or HOMAIR $\geq 1.775$ ) showed no significant association. There was no correlation between testosterone level with severity of depression in both PCOS and non-PCOS groups, although there was significant difference between testosterone level of two groups (PCOS and non-PCOS) (Table 3). No correlation was found between depression score and BMI, HOMA-IR and testosterone level in both PCOS and non-PCOS groups (Table 4). 
Table 3. Comparison of testosterone level in PCOS and control group base on depression Severity*

\begin{tabular}{|l|l|l|l|l|l|l|l|l|l|l|}
\hline \multirow{2}{*}{ Depression score } & \multicolumn{2}{l|}{ Normal } & \multicolumn{2}{l|}{ Mild } & \multicolumn{2}{l|}{ Moderate } & \multicolumn{2}{l|}{ Severe } & \multicolumn{2}{l|}{ Total } \\
\cline { 2 - 12 } & $\mathrm{n}$ & Mean \pm SE & $\mathrm{n}$ & Mean \pm SE & $\mathrm{n}$ & Mean \pm SE & $\mathrm{n}$ & Mean \pm SE & n & Mean \pm SE \\
\hline PCOS & 22 & $77 \pm 28$ & 17 & $65 \pm 22$ & 17 & $58 \pm 19$ & 6 & $80 \pm 28$ & 62 & $68 \pm 28$ \\
\hline Control & 24 & $59 \pm 16$ & 15 & $59 \pm 24$ & 12 & $47 \pm 14$ & 10 & $56 \pm 13$ & 61 & $56 \pm 18$ \\
\hline Total & 46 & $68 \pm 24$ & 32 & $62 \pm 23$ & 29 & $54 \pm 18$ & 16 & $63 \pm 33$ & 123 & $62 \pm 24$ \\
\hline
\end{tabular}

*ANOVA test was used.

Table 4. Correlation of depression score with Clinico-biochemical markers in PCOS and non-PCOS women

\begin{tabular}{|l|l|l|l|l|l|}
\hline Groups & Fearson Correlation & 0.06 & -0.21 & 0.21 & -0.16 \\
\hline \multirow{4}{*}{ PCOS } & HOMA-IR & BMI & Testosterone \\
\cline { 2 - 6 } & p-value & 0.71 & 0.09 & 0.1 & 0.31 \\
\cline { 2 - 6 } & Number & 62 & 62 & 62 & 62 \\
\hline \multirow{4}{*}{ Non-PCOS } & Pearson Correlation & -0.16 & 0.03 & -0.13 & -0.15 \\
\cline { 2 - 6 } & p-value & 0.31 & 0.81 & 0.30 & 0.34 \\
\cline { 2 - 6 } & Number & 61 & 61 & 61 & 61 \\
\hline \multirow{5}{*}{ Total } & Pearson Correlation & 0.08 & -0.11 & 0.04 & -0.16 \\
\cline { 2 - 6 } & p-value & 0.4 & 0.23 & 0.65 & 0.15 \\
\cline { 2 - 6 } & Number & 123 & 123 & 123 & 123 \\
\hline
\end{tabular}

\section{Discussion}

In this study, $64.5 \%$ of PCOS subjects and $60.7 \%$ of the control group had some degree of depression that is not significant. Controversy in the prevalence of depression among PCOS women was reported in different studies (3, 5 , 6, 9). Although in the study performed by Rahiminejad in Hamedan, Iran, 31.7\% of PCOS persons had depression (17), but their study had no control group. In our study, prevalence of depression on both groups is high. Last systematic review and meta-analysis revealed a current prevalence of $4.1 \%$ for major depressive disorder in Iran (general population), and women are more likely to have major depressive disorder, approximately 1.95 times more than men (18). We did not evaluate major depressive disorder in our study, based on the Diagnostic and Statistical Manual IV (DSM-IV) which is another description of depression. Our result is similar to the study by Bhattacharya (6) in which $64.1 \%$ of PCOS women had depressive disorders but in the mentioned study, prevalence of depression among PCOS was significantly higher than the non-PCOS group. This means that prevalence of depression in all of our study population is high. Factors such as economic problems, education level, employment and social limitations, especially for girls are possible factors for high depression among women. The relationship between depression and PCOS is likely complex, and therefore, we considered a variety of factors in our analysis. We did not find any significant association between FBS, HOMA-IR, and testosterone level with depression severity. Also, there is no correlation between BMI and depression score in the present study. Our findings support the previous studies that obesity is not a risk factor for depression in patients with PCOS (17). A systematic review and metaanalysis between women with PCOS and healthy controls revealed that higher depression scores in patients with PCOS, is associated with Body Mass Index (19). The relationship between biochemical markers in women with polycystic ovary syndrome and depression is controversial. The relationship between depression and insulin resistance, has been examined in small studies which have shown different results $(3,6,17,20,21)$. Depression is associated with high cortisol level and increased sympathetic nervous system activity and low serotonin in the central nervous system. These features are also common in insulin resistance state (20). We found no association between HOMA-IR (Marker of insulin resistance) and depression score in PCOS and non-PCOS persons. However, it is necessary to evaluate the relationship between HOMA-IR and depression in PCOS persons in future studies with large sample size. Also, regular exercise and using drugs such as metformin may be a potential confounder of the relationship between depression and insulin resistance that were neglected in our study. Association between depression and testosterone level has been evaluated in several studies with conflicting results $(6,8,10,17)$. In the present study, there is no correlation between testosterone level and depression in PCOS and non-PCOS women and all of the population. The result of Barry showed that women with PCOS had low matching with stress than in subfertile groups, and had more quality of life problems related to the disease manifestations, but no correlation was found between testosterone level and mood disturbance in their study (22). Weber showed that correlation of serum testosterone is significant only in women with severe depression (23). Depression and the severity of depressive symptoms were positively correlated with dehydroepiandrosterone sulfate levels, not testosterone levels in an IRAK study (8). Hypothalamic-pituitary adrenal system dysregulation has an important role in the pathophysiology of 
depression that is reflected by an increased secretion of adrenal hormones, especially cortisol and dehydroepiandrosterone (23). Further research for assessment relationship between depression and testosterone level in a large sample population may be necessary. Also, measurement of dehydroepiandrosterone sulfate may be superior to the testosterone level.

\section{Limitations and suggestions}

This study has several limitations. Cross-sectional design and the small number of participants and few clinical/biochemical parameters investigated in this study are limitations. Also, identification of depression risk by BDI-FS, rather than through the use of a structured clinical interview is another limitation of our study. Because depression has fundamental impairments, it not only influences the performance of daily tasks, but also can lead to fatal acts such as suicidal behavior, study with large sample size and in a community base rather than out-patient clinic for predicting true prevalence of depression in our region may be helpful and necessary. Also, a randomized controlled trial investigating whether depression severity in PCOS women improves along with successful treatment is helpful.

\section{Conclusions}

Prevalence and risk of depression is significantly high in women in this study. We failed to show an association between any of the clinico-biochemical markers and depression severity. No explanation for this could be derived from the selected parameters in the present study. But "absence of evidence is not evidence of absence" (24). Prospective investigation, in larger cohorts in this field, may be helpful.

\section{Acknowledgments:}

The authors thank Shahid Sadoughi University of Medical Sciences, Yazd, Iran for the financial support of this research project (research project no. 2435) and we are very grateful to Masoumeh Mohajeri for assistance with data collection and all the patients who were involved.

\section{Conflict of Interest:}

There is no conflict of interest to be declared.

\section{Authors' contributions:}

All authors contributed to this project and article equally. All authors read and approved the final manuscript.

\section{References:}

1) Kerchner A, Lester W, Stuart SP, Dokras A. Risk of depression and other mental health disorders in women with polycystic ovary syndrome: a longitudinal study. Fertility and sterility. 2009; 91(1): $207-12$. doi: 10.1016/j.fertnstert.2007.11.022. PMID: 18249398 .

2) Dokras A. Mood and anxiety disorders in women with PCOS. Steroids. 2012; 77(4): 338-41. doi: 10.1016/j.steroids.2011.12.008. PMID: 22178257.

3) Rasgon NL, Rao RC, Hwang S, Altshuler LL, Elman S, Zuckerbrow-Miller J, et al. Depression in women with polycystic ovary syndrome: clinical and biochemical correlates. Journal of affective disorders. 2003; 74(3): 299-304. doi: 10.1016/S0165-0327(02)00117-9.

4) Eshre TR, Group A-SPCW. Revised 2003 consensus on diagnostic criteria and long-term health risks related to polycystic ovary syndrome. Fertility and sterility. 2004; 81(1): 19-25. doi: 10.1016/j.fertnstert.2003.10.004.

5) Deeks AA, Gibson-Helm ME, Teede HJ. Anxiety and depression in polycystic ovary syndrome: a comprehensive investigation. Fertility and sterility. 2010; 93(7): 2421-3. doi: 10.1016/j.fertnstert.2009.09.018. PMID: 20117778 .

6) Bhattacharya SM, Jha A. Prevalence and risk of depressive disorders in women with polycystic ovary syndrome. Fertil Steril. 2010; 94: 357-9. doi: 10.1016/j.fertnstert.2009.09.025. PMID: 19896652.

7) Coffey S, Bano G, Mason HD. Health-related quality of life in women with polycystic ovary syndrome: a comparison with the general population using the Polycystic Ovary Syndrome Questionnaire (PCOSQ) and the Short Form-36 (SF-36). Gynecological endocrinology. 2006; 22(2): 80-6. doi: 10.1080/09513590600604541. PMID: 16603432.

8) İrak L, Çinar Yavuz H, Ayçiçek Doğan B, Mete T, Berker D, Güler S. Depression, anxiety, and their relation with clinical parameters and androgen levels in hirsute women. Turkish Journal of Medical Sciences. 2016; 46(2): 245-50. doi: 10.3906/sag-1405-72. PMID: 27511480. 
9) Rassi A, Veras AB, dos Reis M, Pastore DL, Bruno LM, Bruno RV, et al. Prevalence of psychiatric disorders in patients with polycystic ovary syndrome. Comprehensive psychiatry. 2010; 51(6): 599-602. doi: 10.1016/j.comppsych.2010.02.009. PMID: 20965306.

10) Weiner CL, Primeau M, Ehrmann DA. Androgens and mood dysfunction in women: comparison of women with polycystic ovarian syndrome to healthy controls. Psychosom Med. 2004; 66(3): 356-62. PMID: 15184695.

11) Bazarganipour F, Ziaei S, Montazeri A, Foroozanfard F, Kazemnejad A, Faghihzadeh S. Psychological investigation in patients with polycystic ovary syndrome. Health and quality of life outcomes. 2013; 11(1): 141.

12) Beck AT, Rial WY, Rickels K. Short from of depression inventory: cross validation. Psychological Reports. 1974; 34: 1184-6. PMID: 4424377.

13) World Health Organization. Obesity: preventing and managing the global epidemic. World Health Organization; 2000: 894 .

14) Antuna-Puente B, Disse E, Rabasa-Lhoret R, Laville M, Capeau J, Bastard JP. How can we measure insulin sensitivity/resistance? Diabetes Metab. 2011; 37: 179-88. doi: 10.1016/j.diabet.2011.01.002. PMID: 21435930.

15) Wallace TM, Levy JC, Matthews DR. Use and abuse of HOMA modeling. Diabetes Care. 2004; 27: 148795. doi: 10.2337/diacare.27.6.1487. PMID: 15161807.

16) Esteghamati A, Ashraf H, Khalilzadeh O, Zandieh A, Nakhjavani M, Rashidi A, et al. Optimal cut-off of homeostasis model assessment of insulin resistance (HOMA-IR) for the diagnosis of metabolic syndrome: third national surveillance of risk factors of non-communicable diseases in Iran (SuRFNCD-2007). Nutr Metab (Lond). 2010; 7: 26. doi: 10.1186/1743-7075-7-26. PMID: 20374655, PMCID: PMC2857836.

17) Rahiminejad ME, Moaddab A, Rabiee S, Esna-Ashari F, Borzouei S, Hosseini SM. The relationship between clinicobiochemical markers and depression in women with polycystic ovary syndrome. Iran $\mathrm{J}$ Reprod Med. 2014; 12(12): 811-6. PMID: 25709638, PMCID: PMC4330661.

18) Sadeghirad B, Haghdoost AA, Amin-Esmaeili M, Shahsavand Ananloo E, Ghaeli G, Rahimi-Movaghar A, et al. Epidemiology of major depressive disorder in Iran: a systematic review and meta-analysis. Int J Prev Med. 2010; 1: 81-91. PMID: 21566767, PMCID: PMC3075476.

19) Barry JA, Kuczmierczyk AR, Hardiman PJ. Anxiety and depression in polycystic ovary syndrome: a systematic review and meta-analysis. Hum Reprod. 2011; 26: 2442-51. doi: 10.1093/humrep/der197. PMID: 21725075.

20) Okamura F, Tashiro A, Utumi A, Imai T, Suchi T, Tamura D, et al. Insulin resistance in patients with depression and its changes during the clinical course of depression: minimal model analysis. Metabolism 2000; 49: 1255- 60. doi: 10.1053/meta.2000.9515. PMID: 11079812.

21) Adriaanse MC, Dekker JM, Nijpels G, Heine RJ, Snoek FJ, Pouwer F. Associations between depressive symptoms and insulin resistance: the Hoorn Study. Diabetologia. 2006; 49: 2874-7. doi: 10.1007/s00125006-0500-4. PMID: 17066302.

22) Barry JA, Hardiman PJ, Saxby BK, Kuczmierczyk A. Testosterone and mood dysfunction in women with polycystic ovarian syndrome compared to subfertile controls. J Psychosom Obstet Gynaecol. 2011; 32(2): 104-11. doi: 10.3109/0167482X.2011.568129. PMID: 21473679.

23) Weber B, Lewicka S, Deuschle M, Colla M, Heuser I. Testosterone, androstenedione and dihydrotestosterone concentrations are elevated in female patients with major depression. Psychoneuroendocrinology. 2000; 25: 765-71. doi: 10.1016/S0306-4530(00)00023-8.

24) Douglas GA, Bland MJ. Statistics notes: Absence of evidence is not evidence of absence. BMJ. 1995; 311(7003): 485. doi: 10.1136/bmj.311.7003.485. 